\title{
Special Section: Religion and Health Response to the COVID-19 Pandemic
}

\author{
Curtis W. Hart ${ }^{1} \cdot$ Harold G. Koenig ${ }^{2,3,4,5}$
}

Accepted: 2 September 2020 / Published online: 12 September 2020

(c) Springer Science+Business Media, LLC, part of Springer Nature 2020

With this Special Section, the Journal presents, as promised, a series of articles describing responses, individual and communal, to the COVID-19 pandemic sweeping the USA and the world. We have endeavored to follow through on our pledge in the June 2020 issue to provide a wide selection of viewpoints and believe that the following articles will do much to expand knowledge about how and why religion and religious communities have contributed to protection from and knowledge about COVID-19 and its continuing spread. Enlightened by science, this Special Section describes not only the present condition of the disease but also the way forward in the direction of healing of persons and provision of resources for the mending of the social fabric. Eleven of the articles in this Special Section have already been recognized as among the most noteworthy in the field through their selection by the Institute for the Bio-Cultural Study of Religion for inclusion in their Research Review.

From the number of submissions yet to be fully reviewed, we are aware that the Journal continues to receive and welcome submissions on this topic. COVID-19 is not going away nor is our determination to present articles of value across cultures. In this Special Section, we seek to provide a platform for the sharing of knowledge and expertise having currency in the market place of ideas and also to serve as a source for reflection for future researchers and historians during this painful epoch. Future leaders and scholars may ask of religious communities in the COVID-19 era and those who did research: What were they thinking, what did they know, and what

Harold G. Koenig

harold.koenig@duke.edu

Curtis W. Hart

cuh9001@med.cornell.edu

1 Division of Medical Ethics, Departments of Medicine and Psychiatry, Weill Cornell Medicine, New York City, NY, USA

2 Department of Psychiatry and Behavioral Sciences, Department of Medicine, Duke University Medical Center, Durham, NC, USA

3 Department of Medicine, King Abdulaziz University, Jeddah, Saudi Arabia

4 School of Public Health, Ningxia Medical University, Yinchuan, People's Republic of China

5 Shiraz University of Medical Sciences, Shiraz, Iran 
did they do? Did they make a difference and why? We trust that the following pages will have much to say about these questions. They are examined here in a variety of geographical locations and professional settings. Their responses come through voices speaking with candor, compassion, and credibility about the ongoing pandemic crisis. Their impact, we hope, will be both immediate and lasting.

\section{Regular Journal Articles}

We note that the first article in the section for our Regular Journal Articles, Knowledge, Attitudes, and Interactions with Chaplains and Nursing Staff Outcomes, authored by Drs. Tara Liberman and Andzej Kozikowski and their colleagues of the Department of Medicine, North Well Health, Manhasset, New York, has been selected by the Association for Clinical Pastoral Education as its Research Article of the month. We congratulate Drs. Liberman and Kozikowski and their co-authors for this notable accomplishment.

Publisher's Note Springer Nature remains neutral with regard to jurisdictional claims in published maps and institutional affiliations. 